\title{
Progress and Perspectives for Atomic-Resolution Electron Microscopy
}

\author{
David J. Smith
}

Department of Physics and Astronomy and Center for Solid State Science, Arizona State University, Tempe, AZ 85287

The atomic-resolution capabilities of the electron microscope, which first became widely accessible in the 1980s, have had a major impact across many disciplines [1]. With carefully prepared samples and correct operating conditions, assisted by quantitative image recording and processing, image characteristics can be interpreted directly in terms of individual atomic columns. With improvements in hardware and special attention to microscope environment, resolving powers close to or exceeding the one Ångstrom $(0.1 \mathrm{~nm})$ barrier have been achieved by the latest generation of high-voltage HREMs [2-4]. Similar performance levels have been reached by dedicated scanning and conventional medium-voltage instruments [5,6], while sub-Ångstrom electron microscopy to resolutions of close to $0.8 \mathrm{~nm}$ has since been achieved using exit-wave retrieval [7, 8]. Meanwhile, after a relatively dormant period during much of the 1990s, the last several years have witnessed a veritable explosion of further instrumentation hardware, including the successful implementation of aberration correctors for both conventional [9] and scanning [10] TEMs, as well as the design of electron monochromators which provide reduced energy spread and hence improved source coherence [11]. These latest developments have generated great interest and enthusiasm within the microscopy community, as well as attracting much attention from the broader materials community, especially for potential applications related to the rapidly emerging fields of nanoscience and nanotechnology.

All electron lenses suffer from performance-limiting aberrations that must be removed in order to attain theoretical resolution limits. Two-fold astigmatism and third-order axial coma can be corrected manually by an experienced operator but for improved reliability and greater accuracy, online computer control or 'autotuning' of the microscope based, for example, on automated diffractogram analysis is recommended [12]. With the advent of aberration correctors, digital acquisition via slowscan CCD cameras has become indispensable. Computer analysis and control of corrector power supplies is essential for determining and then correcting most aberrations up to fourth order, including three-fold objective lens astigmatism and spherical aberration [13, 14]. In the case of the probe-forming lens of the STEM, correction of the aberrations is done using multiple quadrupoleoctopole elements following analysis of a Ronchigram or shadow image obtained from a thin amorphous film $[10,13]$. For conventional TEM imaging, aberration correction is achieved with a double hexapole system and utilizes a tilt series of diffractograms, again relying on the availability of a thin amorphous film [14]. Aberration correction can also be tackled using a posteriori methods, such as exit-wave reconstruction based on through-focal image-series [7, 8]. Similar resolution enhancements have been achieved using atomic-resolution electron holography [15]. Chromatic aberration remains as a serious obstacle to achievement of deep-sub-Ångstrom electron microscopy. However, it appears that the use of a beam monochromator in combination with $\mathrm{Cs}_{\mathrm{s}}$ correction allows the microscope resolution limit for TEM imaging to be enhanced while still retaining useful beam currents at the sample level [16]. Furthermore, a new quadrupole/octopole design has been recently proposed for STEM imaging that should help offset the impact of chromatic aberration and still enable sub- $0.5 \AA$ probes to be obtained [17]. 
Novel types of applications are beginning to emerge following the recent upsurge of instrumentation development described above. With improved resolution, light elements such as carbon, nitrogen, and oxygen can be separately imaged without overlap from neighboring heavier elements [8, 18], and even atomic columns of lithium can be resolved [19]. Defects and interfaces can be studied without the image delocalization that normally complicates interpretation when imaging with a highcoherence FEG electron source $[14,20]$. Negative spherical aberration together with slight overfocus has been shown to yield images with bright-atom contrast, also allowing the degree of oxygenvacancy ordering and oxygen concentrations at twin boundaries to be determined [21]. Despite these promising advances and further intriguing possibilities, it cannot be overlooked, however, that image interpretation for these 'corrected' TEM imaging conditions remains far from intuitive [22]. Image features change extraordinarily rapidly with thickness and defocus, as well as being sensitive to local orientation changes. This extreme sensitivity increases the likelihood of erroneous conclusions about microstructure without extensive input from image simulations. Incoherent Z-contrast imaging with the STEM does not usually suffer from these problems of image interpretation [5]. However, lack of accessibility to lower- $Z$ elements because of low contrast levels represents a major limitation for many structural investigations using this technique. Meanwhile, it remains to be pointed out that serious discrepancies still remain between measured and simulated TEM image contrast levels [23], and inversion of crystal scattering remains an unresolved open challenge [24].

References

[1] D.J. Smith, Rep. Prog. Phys. 60 (1997) 1513.

[2] F. Phillipp, et al., Ultramicroscopy 56 (1994) 1.

[3] H. Ichinose, et al., J. Electron Microscopy 48 (1999) 887.

[4] T. Kawasaki, et al., J. Electron Microscopy 49 (2000) 711.

[5] P.D. Nellist and S.J. Pennycook, Phys. Rev. Lett. 81 (1998) 4156.

[6] D. van Dyck, et al., Ultramicroscopy 64 (1997) 1.

[7] M.A. O'Keefe, et al., Ultramicroscopy 89 (2001) 215.

[8] C. Kisielowski, et al.,Ultramicroscopy 89 (2001) 243.

[9] M. Haider, et al., Ultramicroscopy 75 (1998) 53.

[10] O.L. Krivanek, et al.,Ultramicroscopy 78 (1999) 1.

[11] H.W.Mook and P. Kruit, Ultramicroscopy 81 (2000) 129.

[12] O.L. Krivanek and P.E. Mooney, Ultramicroscopy 49 (1993) 95.

[13] P.E. Batson, et al., Nature 418 (2002) 617.

[14] B. Kabius, et al., J. Electron Microscopy 51 (2002) S51.

[15] A. Orchowski, et al., Phys. Rev. Lett. 74 (1995) 399.

[16] B. Freitag, et al., Ultramicroscopy 102 (2005) 209.

[17] O.L. Krivanek, et al., Ultramicroscopy 96 (2003) 229.

[18] J.L. Hutchison, et al., Ultramicroscopy in press (2005).

[19] Y. Shao-Horn, et al., Nature Materials 2 (2003) 464.

[20] N. Tanaka, et al., J. Electron Microscopy 52 (2003) 69.

[21] C.L. Jia, et al., Science 299 (2003) 870 and Science 303 (2004) 2001.

[22] J.H. Chen, et al., Ultramicroscopy 98 (2004) 81.

[23] A. Howie, Ultramicroscopy 98 (2004) 73.

[24] M. Lentzen and K. Urban, Ultramicroscopy 62 (1996) 89. 